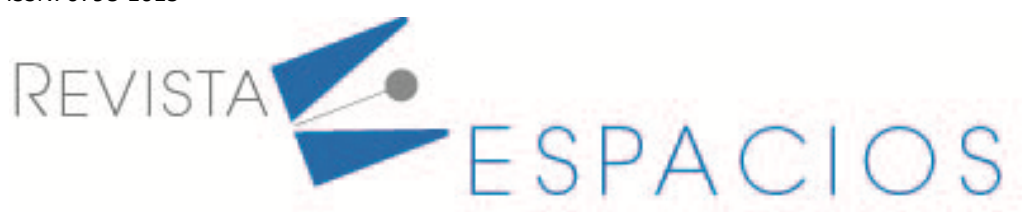

\title{
Capacidades estratégicas en instituciones de educación para el trabajo y desarrollo humano de Bogotá, Colombia
}

\section{Strategic capabilities in educational institutions for job training and human development from Bogota, Colombia}

\author{
BAEZ PALENCIA, Diego A. ${ }^{1,2}$ \\ MARIÑO JIMÉNEZ, Juan P. ${ }^{3}$ \\ PARRA MORENO, Carlos A. ${ }^{4}$ \\ LÓPEZ BARRAZA, Lydia M. ${ }^{5}$
}

\begin{abstract}
Resumen
Este documento analiza capacidades organizacionales y su correspondencia con la postura estratégica, desde una tipología de nivel competitivo. Los datos se obtienen de una muestra de empresas colombianas, sobre los cuales se validó los indicadores que permiten evaluar las variables. Las similitudes buscadas se derivaron de un procedimiento de clústering. Los hallazgos mostraron diferencia en tres grupos para niveles de capacidades, así como relación a estrategias puras e híbridas; explicado por la naturaleza y productos de la industria seleccionada.

Palabras clave: estrategia competitiva, estrategia de negocios, recursos y capacidades, capacidades organizacionales
\end{abstract}

\begin{abstract}
This paper analyses the organizational capabilities and their correspondence with the strategic stance from a typology of the competitive level. Data were gathered from a sample of Colombian companies. To assess the variables, the indicators were validated. The similarities were obtained by using a clustering algorithm. The findings showed different levels of capabilities that were divided into three groups, as well as pure and hybrid strategies for each one. It was explained according to the selected industry's type and products.

keywords: competitive strategy, business strategy, resources-based view, organizational capabilities
\end{abstract}

\section{Introducción}

El mundo empresarial exige a los partícipes ser muy competitivos para lograr posicionarse en los mercados actuales, llenos de gran rivalidad. La tarea del directivo es seleccionar la mejor estrategia posible para crear y sostener su competitividad. Según Castro (2010, p. 248) "no puede ninguna empresa estar compitiendo sin

\footnotetext{
${ }^{1}$ Docente catedrático. Departamento de Estudios Interdisciplinarios. Universidad del Tolima. Ibagué, Colombia. dabaezp@ut.edu.co

${ }^{2}$ Candidato a Doctor en Ciencias Administrativas. Departamento de Ciencias Económico-Administrativas. Universidad Autónoma de Occidente. Culiacán, México.diego.baez@uadeo.mx

${ }^{3}$ Docente investigador. Escuela de Ciencias Económicas y Administrativas. Corporación Universitaria Unitec. juanmarino@unitec.edu.co

${ }^{4}$ Docente de planta. Departamento de Estudios Interdisciplinarios. Universidad del Tolima. Ibagué, Colombia. cfparramgh@ut.edu.co

5 Docente investigadora TC. Departamento de Ciencias Económico-Administrativas. Universidad Autónoma de Occidente. Culiacán, México. lydia.lopez@uadeo.mx
} 
misión, sin objetivos, sin metas y sin las estrategias necesarias para poder lograr esos objetivos, de esta manera es exigente para los propietarios o directivos que dicha decisión sea minuciosamente analizada".

Estas directrices se crean e implementan en todos los niveles de una empresa. Particularmente, las estrategias competitivas se conciben acorde a los recursos y capacidades de la compañía, el entorno, el tipo de mercado, entre otros factores. El estudio de esta tipología de estrategias ha sido abordado por diversos autores, desde la visión temprana de Miles y Snow (1978), Porter (1980, 2013), Miller (1987), Mintzberg (1988) y Kotler (1992, 2012), hasta más de veinte tipologías generadas en desarrollos posteriores; descritos más adelante.

En todas las propuestas de estrategias anteriores está presente el análisis de las capacidades de la empresa como fuente para el alineamiento estratégico; perspectiva generada por Barney (1991) y Grant (1991), y con reciente evolución al concepto dinámico propuesto por Teece, Pisano y Shuen (1997), Teece (2007) y otras derivaciones de este (Vogel \& Güttel, 2013). Dichas capacidades son esenciales para la operacionalización de la estrategia y, por tanto, son de variada índole y cubren las funciones principales al interior de la firma.

Así pues, la presente investigación busca analizar la correspondencia que existe entre las capacidades organizacionales de las empresas, y la postura estratégica adoptada, entendiendo si los rasgos definidos en las conductas son coherentes al conocimiento desarrollado por la compañía. El estudio se realizó sobre una muestra de Instituciones de Educación para el Trabajo y Desarrollo Humano (letdh) de la ciudad de Bogotá, Colombia, encontrando una separación clara entre los niveles de capacidades que representan las estrategias opuestas de prospector y defensor, así como un intermedio en la conducta analizador, que funge como vía de cambio entre las anteriores y sugiere la existencia de estrategias híbridas.

Los hallazgos permiten comprender el actuar de las compañías cuando se tienen circunstancias externas que condicionan el diseño y generación de nuevos productos, caso del mercado estudiado. La postura combinada en su estrategia, explicó los niveles variados en capacidades y la flexibilidad de su proceder. Tales conclusiones permiten a los directivos de las empresas la adecuación de su conocimiento y recursos, según la dinámica de competencia de su dominio de actividad estratégica, y facilita la adopción clara de estrategias viables, que alejen la organización del perfil reactor.

El documento se estructuró en cinco partes. En la primera sección se presenta una revisión de la literatura que soporta el estudio de las variables tratadas. La segunda parte muestra el diseño metodológico empleado. La sección tres expone los resultados en los dos niveles de análisis. Por último se realiza la discusión de los hallazgos y, cierra con las conclusiones, implicaciones prácticas y futuras investigaciones.

\section{Revisión de la literatura}

\subsection{Estrategia competitiva}

El análisis de estrategia ha sido conducido desde diferentes niveles y en variadas aplicaciones (Ansoff, 1979; Porter, 1980; Parnell, 2014; Maia, Di Serio \& Alves, 2015; Maia \& Di Serio, 2017; Farrukh, Meng, Wu \& Nawaz, 2020), así como metodologías de análisis estadístico y difuso (Maia, Di Serio \& Alves, 2015; Baez, Olazabal-Lugo \& Romero-Muñoz, 2020). Algunas de las tipologías de estrategia competitiva (como uno de los niveles de estrategia) se enfocan en la forma de proceder de la organización en un mercado, mientras otras visualizan pensamiento y conducta del directivo de la empresa (Mintzberg, Ahlstrand, \& Lampel, 2010). Respecto a la primera se encuentra Porter $(1980,2013)$, y Miles y Snow (1978) con relación a la última; siendo de las clasificaciones usadas en la literatura (Camisón Zornoza, Garrigós Simón, \& Palacios Marqués, 2007).

Empero, otras tipologías han sido estudiadas como Miller (1987), Mintzberg (1988) y Kotler (1992, 2012) las cuales son recurrentes y extienden los conceptos de las dos precedentes. Los dos primeros realizan una 
derivación de la propuesta de Porter (1980, 2013), particularmente sobre la estrategia de diferenciación. Miller (1987) extiende el nivel estratégico enfocado en la variación de producto sobre aquellos elementos que se relacionan directamente sobre el bien y, de otro lado, los que se generan como complementarios al uso que tiene. Por su parte Mintzberg (1988) desglosa en detalle la propuesta de sus predecesores al diferenciar entre actividades de la mezcla de mercadotecnia. Respecto a Kotler $(1992,2012)$, su propuesta es conductual, similar a Miles y Snow (1978) aunque sin la profundidad de los problemas de ingeniería y administrativos. Centrado en el mercado, Kotler plantea tres comportamientos que conllevan a lograr posición y competencia en un mercado.

Si bien las anteriores son difundidas, varias tipologías de estrategias competitivas pueden encontrarse. Buzze, Gale y Sultan (1975) y Utterback y Abernathy (1975) proponen modelos desde la lógica de perspectiva comportamental, predecesores de Miles y Snow (1978). Hofer y Schendel (1978), Vesper (1979), se acercan más al planteamiento de Porter (1980) desde la arista de la organización industrial. Posteriores a estos dos seminales, Abell (1980), Wissema, Van Der Pol y Messer (1980), Miles (1982), Galbraith y Schendel (1983) y March (1991) realizan estudios empíricos sobre la aplicación conductual de Miles y Snow (1978), derivando de ellos subtipologías de orden conductual; esto respecto a la decisión del directivo.

En cuanto a Porter (1980), su estudio y análisis de campo realizado por Herbert y Deresky (1987), Robinson y Pearce (1988) y Douglas y Rhee (1989) se exponen variaciones o subdivisiones bajo el paradigma microeconómico que determina el proceder de competencia en la industria para lograr una posición. Treacy y Wiersema (1997) plantean una tipología integradora, posterior a su análisis de Miles y Snow (1978) y Porter (1980).

Asimismo, es posible encontrar otras combinaciones híbridas, tanto de Porter $(1980,2013)$ como de Miles y Snow (1978), las cuales no sitúan a la empresa en un extremo de estrategia competitiva, sino más bien en una mezcla entre aquellas que se acercan en sus características. Esto ha sido resultado de investigaciones aplicadas por Claver-Cortés, Pertusa-Ortega y Molina-Azorín (2012), Desarbo \& Grewal (2008) y Anwar y Hasnu (2017).

Entre las nombradas, la propuesta de Miles y Snow (1978) es ampliamente usada en investigaciones sobre estrategia. Su planteamiento determina el cómo una organización piensa para su actuar, atendiendo los problemas de mercado, de operación y administrativos. Al ser flexible la categorización, permite clasificar a la organización en una de las vertientes de la tipología, siendo aplicable a empresas con lucro o sin lucro, de cualquier giro industrial, e independiente de la mezcla de bienes que genere (Blackmore \& Nesbitt, 2013; Bouhelal \& Kerbouche, 2016; Lin, Tsai, \& Wu, 2014).

El trazado de Miles y Snow (1978) gira alrededor del ciclo adaptativo de la firma a su entorno, ensanchando las ideas de Chandler (1962), Thompson (1967), Cyert y March (1963) y Child (1972). Aunque el enfoque Porteriano también considera el ambiente para la formulación estratégica (Porter, 1980, 2013), éste propone una visión microeconómica para el movimiento en el mercado al determinar si sacar provecho del precio o la cantidad. Mientras que, por su parte, Miles y Snow (1978) lo exponen desde el pensamiento y dirección del gerente para abarcar o mantener un espacio dentro de la dinámica del mercado.

En ese orden de ideas, la propuesta de Miles y Snow (1978) se fundamenta en la existencia de cuatro tipos estratégicos de organizaciones: defensores, analizadores, prospectores y reactores. Cada una de ellas tiene su propia y única forma para relacionarse con su mercado, y una configuración particular en lo que a tecnología, estructura y procesos se refiere; exceptuando al perfil reactor en el que existen inconsistencias entre su estrategia y configuración.

A esto último, Miles y Snow (1978) señalan que la postura reactor es una estrategia fallida en el sentido que dichas organizaciones no logran determinar una respuesta al problema de mercado, ni al de ingeniería, es decir, la definición del dominio producto con segmento; tampoco al problema administrativo, que le permite tener una 
estructura organizacional para moverse en una industria en el dominio determinado. Por tanto, Miles y Snow (1978) no excluyen a empresas que proceden en esta forma, pero sólo incluyen como estrategias de éxito al prospector, analizador y defensor. El cuadro 1 resume sus características.

\section{Cuadro 1}

Tipos de estrategias de Miles y Snow

\begin{tabular}{|c|c|c|}
\hline Estrategia & Característica principal & Consecuencias \\
\hline Defensor & $\begin{array}{l}\text { La alta dirección no busca } \\
\text { nuevas oportunidades fuera de } \\
\text { su negocio. Trabajan en } \\
\text { mercados estables. }\end{array}$ & $\begin{array}{l}\text { Produce un conjunto limitado de productos y controla nichos seguros } \\
\text { en sus industrias. Crece a través de penetración de mercado. } \\
\text { Ajustes mínimos en tecnología, estructura o métodos, sin invertir en } \\
\text { innovación. } \\
\text { Se concentra en eficiencia y la gestión de los costos. Monitoreo bajo } \\
\text { del entorno. }\end{array}$ \\
\hline Prospector & $\begin{array}{l}\text { Continuamente busca nuevas } \\
\text { oportunidades de desarrollo de } \\
\text { productos y nuevos mercados. } \\
\text { Promueve procesos de } \\
\text { innovación y desarrollo. Son } \\
\text { descentralizadas. }\end{array}$ & $\begin{array}{l}\text { Busca ser pionera en su industria. Propicia cambios de paradigmas ante } \\
\text { los que responde el mercado. } \\
\text { No escatima esfuerzos en el uso de recursos económicos. Es flexible } \\
\text { para moverse en el entorno dinámico. }\end{array}$ \\
\hline Analizador & $\begin{array}{l}\text { Postura ecléctica pues en } \\
\text { entornos estables opera de } \\
\text { forma rutinaria y eficiente, y en } \\
\text { contextos turbulentos sigue a } \\
\text { los competidores más } \\
\text { innovadores. }\end{array}$ & $\begin{array}{l}\text { Se mueve hacia nuevos productos o mercados cuando la viabilidad ha } \\
\text { sido comprobada por los prospectores. Minimiza riesgos y maximiza } \\
\text { beneficios. } \\
\text { En entornos estables trabaja con estructuras y procesos formales, } \\
\text { mientras en ambientes cambiantes, vigila los competidores para } \\
\text { adaptarse rápidamente. }\end{array}$ \\
\hline Reactor & $\begin{array}{l}\text { No desarrolla características } \\
\text { organizativas con las cuales } \\
\text { pueda responder de forma } \\
\text { efectiva y sistemática a su } \\
\text { entorno. }\end{array}$ & $\begin{array}{l}\text { Se limita a detectar señales emanadas por parte de los mejores de la } \\
\text { industria y opta por una u otra postura estratégica. } \\
\text { Presentan rendimientos inferiores a los anteriores. - Anwar y Hasnu } \\
\text { (2017) han encontrado resultados contrarios para esta postura } \\
\text { estratégica -. }\end{array}$ \\
\hline
\end{tabular}

Fuente: Elaboración propia con base en Miles y Snow (1978)

\subsection{Capacidades organizacionales}

El enfoque de recursos y capacidades es un marco de estudio con influencia en la comprensión de la gestión estratégica (Barney, Wright, \& Ketchen, 2001). Su desarrollo los dibuja como determinantes del rendimiento que puede lograr la empresa (Amit \& Schoemaker, 1993); siendo así planteados por Barney (1991) y Grant (1991) como respuesta a esas variaciones de resultado, al entendido que estos permiten el adelanto de estrategias para alcanzar un ventaja competitiva.

Los recursos, que deben ser únicos, de difícil imitación y obtención, permiten el mantenimiento de la ventaja sostenible (O'Shannassy, 2008). Sin embargo, la forma de operación de la empresa debe estar alineada con las condiciones de mercado, razón por la cual dichos recursos deben estar en continua adaptación conforme el entorno lo exige.

A tal efecto, desarrollos posteriores condujeron a dos vertientes: la visión basada en conocimiento (Grant, 1996; Zander \& Kogut, 1995), y el enfoque de capacidades dinámicas (Danneels, 2008, 2010; Teece et al., 1997; TorresBarreto, Noel, Meza-Ariza \& Muñoz, 2015). Las dos perspectivas, más que controvertirse, se complementan, pues el conocimiento que posee el órgano social, permite el desarrollo del segundo enfoque, lo mantiene y evoluciona. 
Esas capacidades dinámicas son definidas como la habilidad para redistribuir, modificar, renovar y reproducir la base de recursos de la compañía, así como de moldear sus capacidades organizacionales, todo eso apuntando hacia al mejor ajuste con el entorno (Ambrosini \& Bowman, 2009; Wang \& Ahmed, 2007; Zahra, Sapienza, \& Davidsson, 2006). Entonces, las capacidades son de relevancia para el desarrollo estratégico de la firma, fuente de ventaja competitiva (Barney, 2002; Suárez Hernández \& Ibarra Mirón, 2002), y conductores de rendimiento, basado en la alineación con el ambiente (Parnell, 2011; Parnell \& Hershey, 2005; Parnell, Long, \& Lester, 2015).

Si bien varios estudios han abordado las capacidades y su resultado en la empresa, existe una diferencia en el tipo o especificidad que es considerada. Conant, Mokwa y Varadarajan (1990) analizan las capacidades de marketing o empresariales, administrativas, y tecnológicas o producción, siendo conceptuadas según las problemas organizacionales que plantean Miles y Snow (1978); similar tratamiento realizan Blackmore y Nesbitt (2013).

Desarbo, Benedetto, Song y Sinha (2005) agregan las capacidades de enlace con el mercado, y de tecnologías de información. La primera de ellas considera las relaciones con los miembros del canal e industria, como parte del monitoreo e incertidumbre del entorno en el que se encuentra la organización; esto es analizado en Parnell (2011), Parnell et al (2015), Parnell y Brady (2019), Ynzunza e Izar (2013) y Chryssochoidis, Dousios y Tzokas (2016). Mientras la segunda corresponde al uso y gestión de sistemas de información para el control interno y externo de la firma; como propuesto por Day (1994) en lo que denomina conductoras de mercado.

Aunque este fundamento organizacional varía en su forma de análisis, coinciden los autores en que los recursos y capacidades son antecedentes focales para el rendimiento que alcanza la firma, así como elemento de relación con la estrategia. La distancia que exista entre la configuración de estas y la postura estratégica de alineación con el entorno, representa la diferencia en el resultado que pueda lograr la empresa (Claver-Cortés, PertusaOrtega, \& Molina-Azorín, 2011; Claver-Cortés et al, 2012; Jusoh \& Parnell, 2008).

\section{Metodología y validación}

\subsection{Datos y muestra}

Esta investigación consideró el sector de servicios, particularmente el mercado de educación para el trabajo y desarrollo humano en la ciudad de Bogotá, Colombia. La población fue de 446 instituciones, con registro emitido por la Secretaría de Educación. Se excluyeron aquellas que no ofertaran al menos un programa de formación técnica, obteniendo un total de 336 instituciones (letdh) a cierre de 2017, según reporte del Ministerio de Educación Nacional (2017).

Se obtuvo una muestra de 124 instituciones, bajo un muestreo estratificado con confianza de $95 \%$ y error muestral 0,07, siendo 71 letdh en el grupo con mezcla de productos baja, y 53 para las que ofertan dos o más titulaciones; estas cantidades en estrato sólo se consideraron para el análisis descriptivo, y el conjunto global fue utilizado en la metodología. Los instrumentos fueron aplicados de manera guiada con el directivo de la letdh. De las respuestas obtenidas, seis de ellas no fueron contempladas para análisis por información faltante.

\subsection{Medición del perfil estratégico}

Para la recolección de datos se utilizó un instrumento adaptado de Segev (1987a, 1987b), Conant, Mokwa y Varadarajan (1990) y Jusoh y Parnell (2008), con objeto de establecer la estrategia competitiva que sigue la firma. Este cuestionario evalúa indicadores derivados de los problemas de mercado, ingeniería y administrativo, es decir, sobre la mezcla de producto, gestión del segmento y decisiones organizacionales. El instrumento se muestra en el anexo 1. 
Teniendo en cuenta los cambios en el instrumento, así como el uso en un contexto diferente al originalmente concebido, se analizó su validación. Para la variable estrategia, dada la similitud en los indicadores evaluados y su diferente orientación según la postura, se utilizó una valoración de expertos hasta alcanzar concordancia entre jueces; para este caso se requirió de dos rondas de evaluación o juicio.

Los jueces fueron seleccionados según experiencia en investigación o en la práctica empresarial, educación, conocimiento lingüístico y disponibilidad para el ejercicio (McGartland Rubio, Berg-Weger, Tebb, Lee, \& Rauch, 2003; Skjong \& Wentworth, 2000; Summers, Williamson, \& Read, 2004). Se convocó a quince expertos pero se obtuvo respuesta de nueve evaluadores para las dos rondas de juicio, siendo una cantidad adecuada para la validación (Escobar-Pérez \& Cuervo-Martínez, 2008; Hyrkäs, Appelqvist-Schmidlechner, \& Oksa, 2003).

Dicha evaluación implicó la valoración del experto sobre el indicador que evalúa la variable estrategia competitiva, así como sobre cada pregunta respecto a los criterios de claridad, esto es, la comprensión según su sintáctica y semántica, y la coherencia, a saber, la relación lógica con la dimensión a medir. Además, se indagó la suficiencia de los elementos que componen cada postura, como referencia que permite obtener una medición completa de la variable.

Ahora bien, diferentes medidas de concordancia, en escalas nominales $u$ ordinales, pueden ser utilizadas para una evaluación del tipo indicado (Escobar-Pérez \& Cuervo-Martínez, 2008). En este análisis se aplicó el estadístico Kappa de Fleiss (Fleiss, 1971), siendo una extensión de la medida Kappa de Cohen (Cohen, 1960), pues permite aplicar la medición cuando se tiene más de dos jueces en el procedimiento de evaluación (Fleiss, 1971).

Para la primera ronda de evaluación (juicio) se obtuvo un índice Kappa de Fleiss de 0.4159, requiriendo adecuación pues no alcanzó el nivel moderado o mayor (Fleiss, 1971; Landis \& Koch, 1977). El segundo juicio logró un acuerdo de 0.6137 , pasando así el nivel substancial y aceptando la validez del instrumento.

Además, se analizó la fiabilidad de escala con objeto de comprender si la medida obtenida es coherente según los respondientes. Con el uso del Alfa de Cronbach en cada dimensión del instrumento, se obtuvo niveles aceptables (George \& Mallery, 2016) en todas las posturas (defensor 0.74, prospector 0.78, analizador 0.76, y reactor 0.69).

\subsection{Medición de las capacidades organizacionales}

En cuanto a la medición de la variable capacidades se aplicó un instrumento guiado, que fue adaptado a partir de Desarbo et al (2005). Considerando que el instrumento contempla cinco variables diferentes (ver anexo 2) y con indicadores distintos, se realizó un análisis factorial confirmatorio para conocer si cada conjunto de seis indicadores mide, en el grupo de empresas, las capacidades (latentes) de marketing, enlace de mercado, tecnología de información, tecnológica u operación, y de gestión.

Para la capacidad de marketing se incluyeron indicadores de conocimiento de clientes y competidores, de actividad de segmentación, y de integración de la mezcla de mercadotecnia. En la capacidad de enlace de mercado se considera la creación y gestión de relaciones con stakeholders (proveedores, canales y clientes). La capacidad de tecnología de información se analiza desde el soporte que brindan los sistemas de información para el desarrollo de producto, la creación e integración del conocimiento en la empresa, y la comunicación. Con relación a la capacidad tecnológica u operación fueron tenidos en cuenta los procesos de desarrollo y fabricación del producto o servicio y el control de la calidad, y la predicción de cambios y generación de innovación en tecnología y procesos. Para la última capacidad, de gestión, incluye elementos logísticos, financieros, de planificación de recursos humanos y de marketing y estratégica, y anexando planeación y seguimiento a la estrategia. 
Todos los indicadores (reactivos), en su relación a la latente, fueron significativos a un $\alpha=0.01$ (ver figura 1). Así mismo, varios índices mostraron buen ajuste del modelo. La medida de ajuste comparativo (CFI) fue de 0.921, el coeficiente de Tucker-Lewis fue de 0.913, y el error cuadrático medio de aproximación (RMSEA) fue de 0.057 ubicándose en el intervalo de ajuste razonable (Browne \& Cudeck, 1992).

Figura 1

Estructura indicadores y capacidades organizacionales

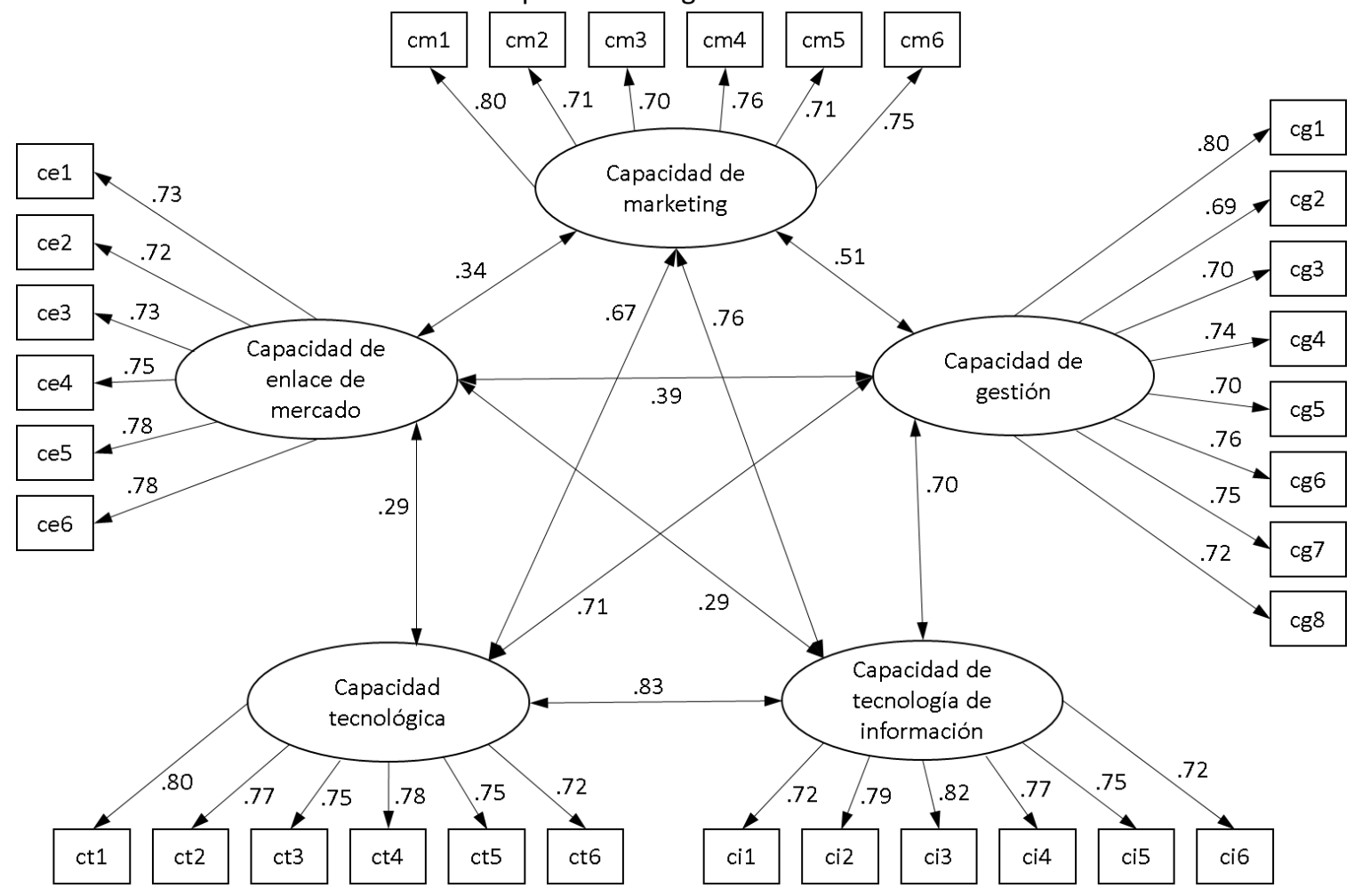

\begin{tabular}{|ll|}
\hline Nota de abreviaturas: & $\mathrm{cm} \#=$ indicador número \# en la capacidad de marketing \\
& $\mathrm{ce \#}=$ indicador número \# en la capacidad de enlace de gestión \\
& $\mathrm{ct \#}=$ indicador número \# en la capacidad tecnológica \\
& $\mathrm{ci \#}=$ indicador número \# en la capacidad de tecnología de información \\
& $\mathrm{cg} \#=$ indicador número \# en la capacidad de gestión \\
\hline
\end{tabular}

Fuente: elaboración propia

Adicional, el alfa de Cronbach mostró resultados aceptables en cada latente identificada: capacidad de marketing 0.87, enlace de mercado 0.88 , tecnología de información 0.89 , tecnológica 0.88 y gestión 0.902 .

Para este análisis se utilizó AMOS versión 24. Si bien algunos autores plantean un tamaño de muestra de al menos 200 observaciones para utilizar el enfoque basado en covarianza (Catena, Ramos, \& Trujillo, 2003; Hair, Black, Babin, \& Anderson, 2019; Pituch \& Stevens, 2016) no hay un consenso al respecto. Otros estudios sugieren tamaños por debajo de esa cantidad según el número de parámetros (Astrachan, Patel, \& Wanzenried, 2014; Kline, 2016; Worthington \& Whittaker, 2006). No obstante, analizando la normalidad de los datos, la prueba de Mardia obtuvo un valor-p de 0,1763, asumiendo así esta distribución.

\subsection{Métodos}

Para establecer el perfil estratégico de cada letdh, se aplicó una función de membresía (ecuaciones 1 a 5) que evalúa cada empresa según los indicadores del instrumento y su propensión en algún comportamiento. 


$$
\mathbf{M}=\left\{\begin{array}{lll}
1, & \text { si } & \operatorname{Max}\left\{\theta_{\mathcal{D}}, \theta_{\mathcal{P}}, \theta_{\mathcal{A}}, \theta_{\mathcal{R}}\right\}=\theta_{\mathcal{D}} \\
2, & \text { si } & \operatorname{Max}\left\{\theta_{\mathcal{D}}, \theta_{\mathcal{P}}, \theta_{\mathcal{A}}, \theta_{\mathcal{R}}\right\}=\theta_{\mathcal{P}} \\
3, & \text { si } & \operatorname{Max}\left\{\theta_{\mathcal{D}}, \theta_{\mathcal{P}}, \theta_{\mathcal{A}}, \theta_{\mathcal{R}}\right\}=\theta_{\mathcal{A}} \\
0, & \text { si } & \operatorname{Max}\left\{\theta_{\mathcal{D}}, \theta_{\mathcal{P}}, \theta_{\mathcal{A}}, \theta_{\mathcal{R}}\right\}=\theta_{\mathcal{R}}
\end{array}\right.
$$

donde,

$$
\begin{gathered}
\theta_{\mathcal{D}}=\frac{\sum_{i=1}^{9} r_{i}}{\operatorname{Max}\left\{i_{\theta_{\mathcal{D}}}\right\}} \\
\theta_{\mathcal{A}}=\frac{\sum_{i=18}^{25} r_{i}}{\operatorname{Max}\left\{i_{\theta_{\mathcal{A}}}\right\}}
\end{gathered}
$$

$$
\begin{aligned}
& \theta_{\mathcal{P}}=\frac{\sum_{i=10}^{17} r_{i}}{\operatorname{Max}\left\{i_{\theta_{\mathcal{P}}}\right\}} \\
& \theta_{\mathcal{R}}=\frac{\sum_{i=26}^{31} r_{i}}{\operatorname{Max}\left\{i_{\theta_{\mathcal{R}}}\right\}}
\end{aligned}
$$

siendo,

$$
\begin{gathered}
r_{i}=\text { el valor de respuesta en el indicador } i \\
\mathbf{M}=1=\text { Defensor } \quad \mathbf{M}=2 \text { = Prospector } \quad \mathbf{M}=3=\text { Analizador } \quad \mathbf{M}=0=\text { Reactor }
\end{gathered}
$$

En cuanto a las capacidades, dado que no existe literatura que señale mayor importancia de alguna de esas respecto a las demás, ni tampoco sobre la relevancia de algún indicador, se realizó una agregación a la media para cada variable latente. El resultado es el valor tomado para el posterior procedimiento.

Respecto al tratamiento de los datos se aplicó un análisis de clúster, aplicando el algoritmo de k-means (Witten, Frank, Hall, \& Pal, 2017) - utilizando el software R versión 3.6.1, en este y posteriores análisis -, a fin de determinar el agrupamiento de las empresas según las capacidades organizacionales (Blackmore \& Nesbitt, 2013); estos fueron figurados usando la técnica de caras de Chernoff (1973) que permite hacer representaciones multidimensionales. El resultado del clustering fue aceptado si existía convergencia hasta 10 iteraciones. Se evaluó con un ANOVA y se consideraron pruebas post hoc para cada variable de capacidad.

Así pues, se analizó la afinidad de las características de cada perfil estratégico con los indicadores de la capacidad, determinando un orden de las conductas según las medidas agregadas de las capacidades. Con esto se identificó la estrategia dominante en cada grupo.

\section{Resultados}

\subsection{Descriptivo de estrategia y capacidades organizacionales}

Las estrategias defensor y analizador presentaron la mayor representación en el total de empresas. La situación es similar cuando se considera por estratos según línea de producto (bajo), o más de una (alto) (ver cuadro 2).

Las capacidades, por su parte, se clasificaron en tres intervalos para el análisis. Arriba de la mitad de las letdh poseen un nivel alto (intervalo de calificación $(6,8]$ ), exceptuando para la capacidad tecnológica, en la que su mayoría califica en nivel medio (intervalo $(3,6])$. En el extremo inferior (intervalo $[1,3]$ ) sólo aparece un pequeño reducto de empresas (2,54\%) para la capacidad de marketing, y tecnológica. La figura 2 muestra el descriptivo de frecuencia por niveles en el agregado de las cinco capacidades. 
Cuadro 2

Postura estratégica en las letdh

\begin{tabular}{|c|c|c|c|c|c|c|c|c|}
\hline & & \multicolumn{2}{|c|}{ Estratos } & & & \multicolumn{2}{|c|}{ Estratos } & \multirow[b]{2}{*}{ Total } \\
\hline & Estrategia & ALTO & BAJO & Total & & ALTO & BAJO & \\
\hline Recuento & Analizador & 22 & 25 & 47 & Prospector & 7 & 4 & 11 \\
\hline$\%$ dentro de estrategia & & $46.8 \%$ & $53.2 \%$ & $100.0 \%$ & & $63.6 \%$ & $36.4 \%$ & $100.0 \%$ \\
\hline$\%$ dentro de estrato & & $38.6 \%$ & $41.0 \%$ & $39.8 \%$ & & $12.3 \%$ & $6.6 \%$ & $9.3 \%$ \\
\hline$\%$ del total & & $18.6 \%$ & $21.2 \%$ & $39.8 \%$ & & $5.9 \%$ & $3.4 \%$ & $9.3 \%$ \\
\hline Recuento & Defensor & 27 & 30 & 57 & Reactor & 1 & 2 & 3 \\
\hline$\%$ dentro de estrategia & & $47.4 \%$ & $52.6 \%$ & $100.0 \%$ & & $33.3 \%$ & $66.7 \%$ & $100.0 \%$ \\
\hline$\%$ dentro de estrato & & $47.4 \%$ & $49.2 \%$ & $48.3 \%$ & & $1.8 \%$ & $3.3 \%$ & $2.5 \%$ \\
\hline$\%$ del total & & $22.9 \%$ & $25.4 \%$ & $48.3 \%$ & & $0.8 \%$ & $1.7 \%$ & $2.5 \%$ \\
\hline Recuento & Total & 57 & 61 & 118 & & & & \\
\hline$\%$ del total & & $48.3 \%$ & $51.7 \%$ & $100.0 \%$ & & & & \\
\hline
\end{tabular}

Fuente: Elaboración propia

Figura 2

Capacidades organizacionales en el global de letdh
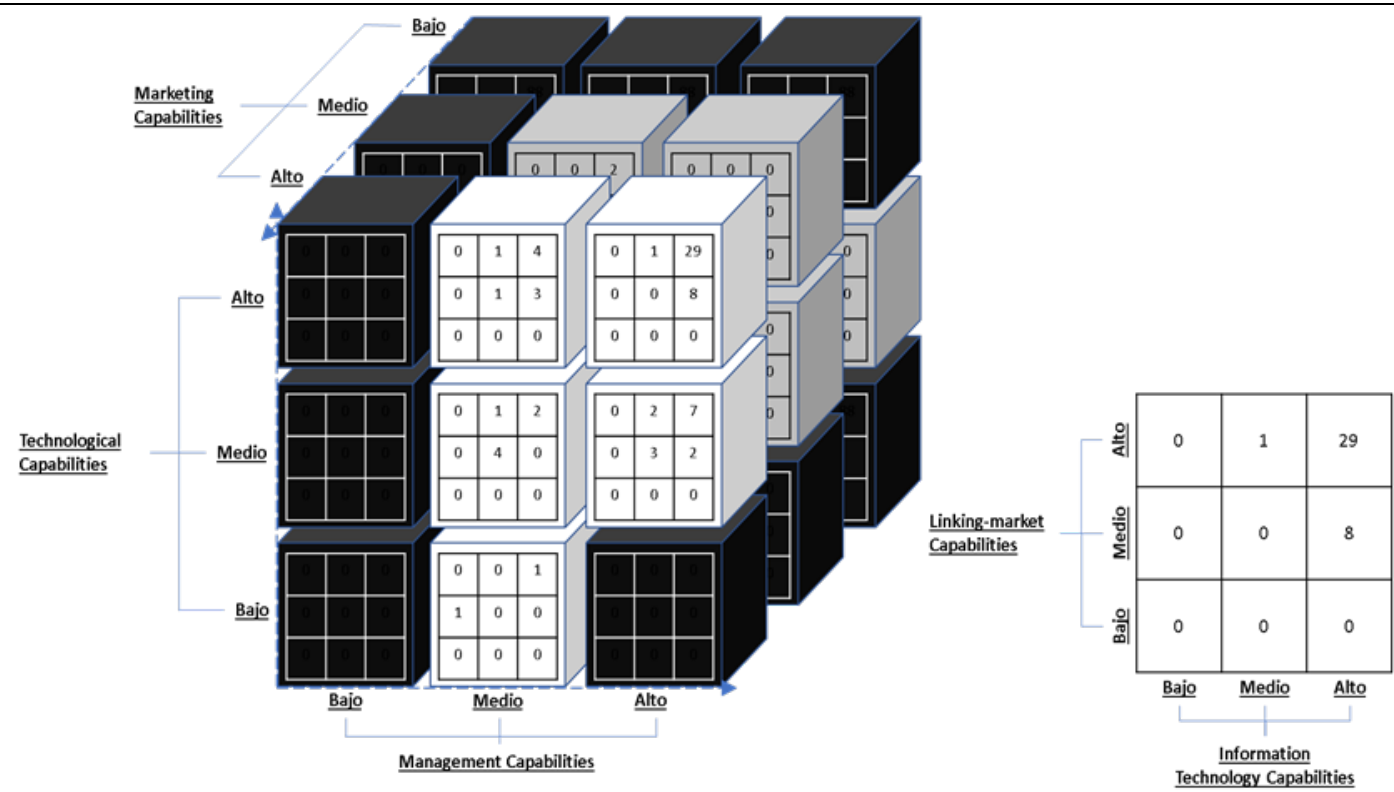


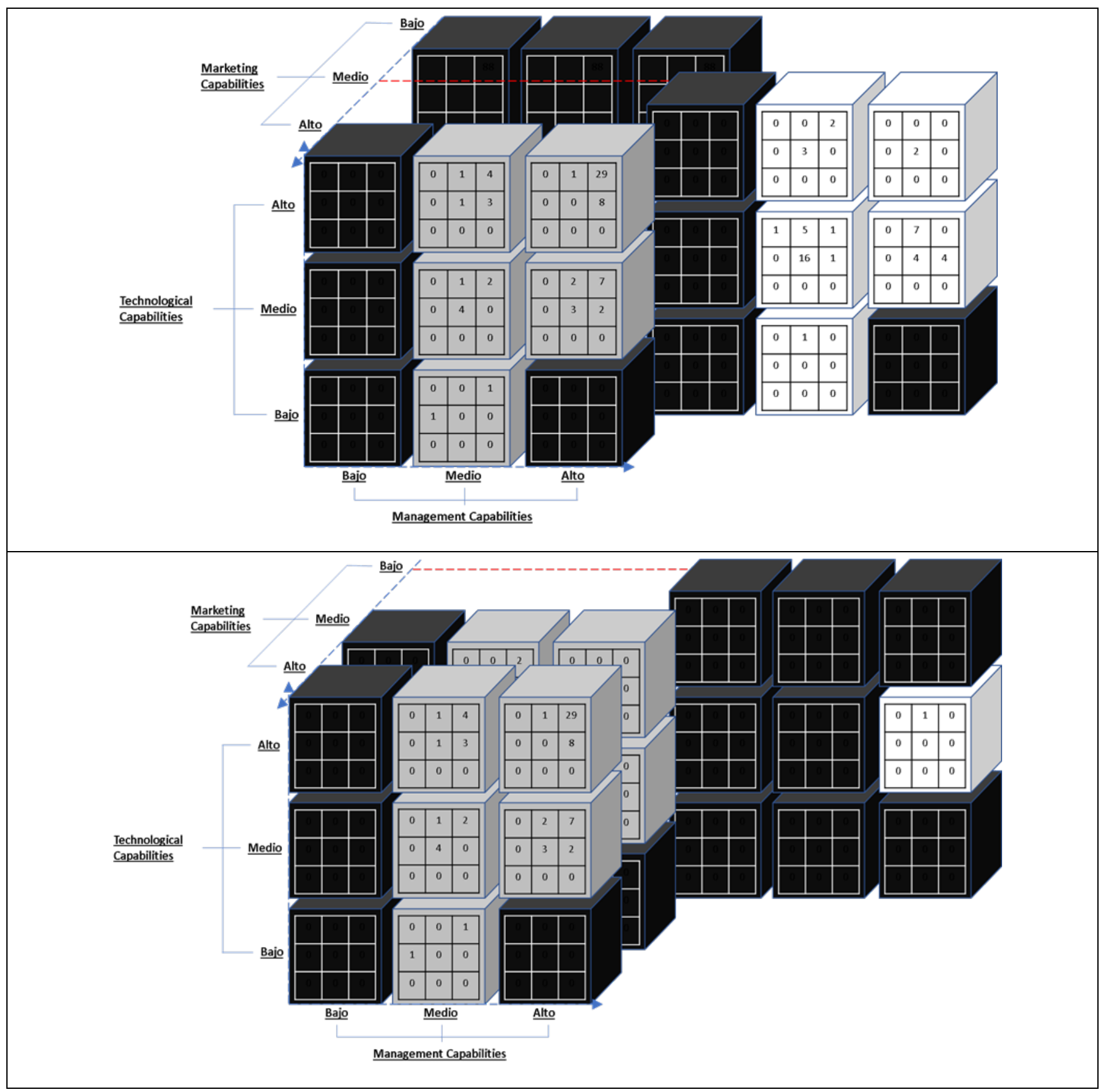

Fuente: elaboración propia

\subsection{Clúster de letdh según sus capacidades organizacionales}

Para el clustering se estipuló como parámetro de agrupamiento un valor de tres. Este guarismo fue determinado al considerar el número de estrategias de la tipología medida; es decir, si se considera la existencia de grupos estratégicos bajo el modelo de Miles y Snow (1978), no deberán ser contados más grupos que las posibles opciones estratégicas disponibles en el análisis, pues justamente eso se desea comprobar. Ahora bien, ya que una de las cuatro opciones es determinada como falla (carencia de dirección administrativa, técnica y de ingeniería) sólo se tienen en cuenta las restantes. El procedimiento restante es dado por distancias a un centroide según las variables del clustering.

Los resultados mostraron que el clúster 1 presentó la mayor agrupación (63 individuos), seguido del conjunto 3 (39) y del grupo 2 (16). Aunque el ANOVA dio cuenta de significancia en cada capacidad (ver cuadro 3), los centros 
de clúster evidencian cercanía entre los grupos 1 y 2 para las capacidades de enlace de mercado, y de gestión; situación que se expone en las pruebas post hoc.

Cuadro 3

ANOVA y pruebas post hoc para clústeres

\begin{tabular}{|l|c|c|c|c|c|}
\hline & & & \multicolumn{3}{|c|}{ Post hoc clúster (valor-p) } \\
\hline & F & Sig. & $\mathbf{1 - 2}$ & $\mathbf{1 - 3}$ & $\mathbf{2 - 3}$ \\
\hline Cap. de marketing & 50.886 & 0.000 & 0.000 & 0.000 & 0.000 \\
\hline Cap. de enlace de mercado & 10.646 & 0.000 & $\mathbf{0 . 4 5 6}$ & 0.001 & 0.000 \\
\hline Cap. de tecnologías de información & 147.352 & 0.000 & 0.000 & 0.000 & 0.000 \\
\hline Cap. tecnológicas & 146.980 & 0.000 & 0.000 & 0.000 & 0.000 \\
\hline Cap. de gestión & 73.944 & 0.000 & 0.000 & 0.000 & 0.000 \\
\hline \multicolumn{2}{|r|}{} \\
\hline
\end{tabular}

Fuente: Elaboración propia

En el comportamiento de las capacidades, visto individualmente, el clúster 3 presentó la mayor calificación (intervalo $(6,8]$ ) en todas las variables. Seguido del grupo 1 donde la dinámica estuvo entre un nivel medio-alto y alto-bajo (intervalo $(4,5,7])$, disminuyendo en capacidades de tecnologías de información, y tecnológica. El clúster remanente obtuvo la menor valoración (intervalo $(3,6])$ aunque no lejana de los demás, siendo las dos últimas capacidades nombradas las situadas en el límite inferior. La figura 3 plasma, de manera multidimensional, estos resultados y facilita apreciar la diferencia en puntuación y hacer el análisis.

Figura 3

Representación de las capacidades organizacionales según clustering

\section{Clúster 1}

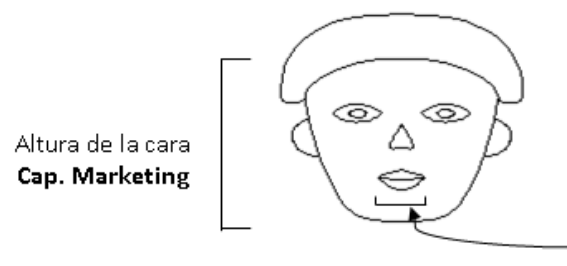

Clúster 2

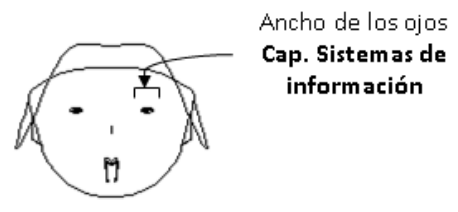

Ancho de la boca

Cap. Gestión

\section{Clúster 3}

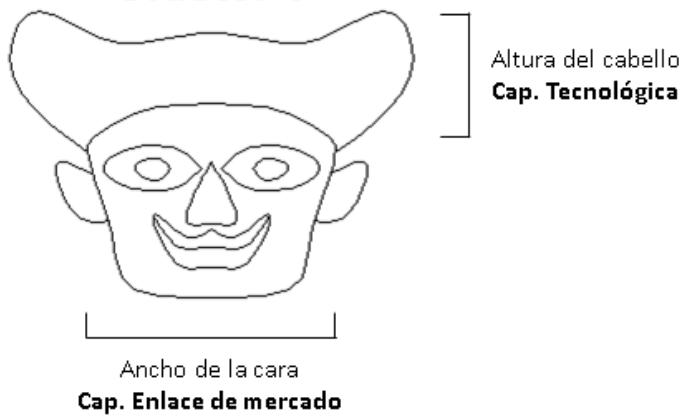

Fuente: elaboración propia

En el diagrama, los rasgos para la capacidad de enlace de mercado no son lejanos entre clúster, lo cual representa la baja diferencia entre la calificación entre grupos, así como en los centros de clúster. En adición, la prueba post 
hoc mostró no significancia en un par en esta variable (ver cuadro 3). En las demás características son notables las distancias.

\subsection{Estrategia en clúster según capacidades}

Considerando lo dispuesto en Miles y Snow (1978) respecto a las características de cada perfil estratégico, detallada su relación al ciclo adaptativo en Conant et al (1990), y posicionando estos en las capacidades organizacionales que soportan la estrategia, planteadas por Desarbo et al (2005), es posible relacionar cada una con mayor o menor propensión al perfil estratégico.

Así pues, considerando lo anterior y teniendo en cuenta los intervalos de las capacidades, se determinó el nivel de medida con mayor frecuencia que refleja el comportamiento de cada capacidad en las estrategias competitivas viables.

Acorde a tal graduación y cruzando con la calificación media obtenida por cada capacidad para cada clúster se encontró la postura estratégica que mejor lo representa, por capacidad y en global (ver cuadro 4).

Cuadro 4

Perfiles estratégicos en clúster según capacidades

\begin{tabular}{|l|c|c|c|}
\hline & Clúster 1 & Clúster 2 & Clúster 3 \\
\hline Cap. de marketing & Prospector / Analizador & Defensor & Analizador \\
\hline Cap. de enlace de mercado & Prospector & Defensor & Analizador \\
\hline Cap. de tecnologías de información & Prospector & Defensor & Analizador / Defensor \\
\hline Cap. tecnológicas & Analizador / Prospector & Analizador & Defensor \\
\hline Cap. de gestión & Prospector & Defensor & Analizador \\
\hline \multicolumn{1}{|c|}{ Postura dominante } & Prospector - Analizador & Defensor & Analizador - Defensor \\
\hline
\end{tabular}

\section{Discusión}

A partir de los resultados es posible derivar algunos hallazgos relevantes. Primero, los indicadores planteados por Desarbo et al (2005) para evaluar cinco capacidades organizacionales, que abarcan las distintas funciones de la empresa, son una medida con validez para establecer el nivel de cada variable latente. Este instrumento ha sido aplicado en industrias y ámbitos geográficos variados (Danneels, 2008; Parnell, 2011; Parnell et al., 2015; Parnell \& Brady, 2019), y se reafirma su adecuación para el sector de servicios, en un entorno latinoamericano.

Por otra parte, en el tipo estratégico que se afilian las letdh, aunque defensor y analizador son las posturas dominantes, prospector es el único perfil con mayor concentración en la comparación entre estratos. Esta estrategia tiene más participación cuando se trata de empresas con varias líneas de producto, frente al conjunto de compañías que opera sobre una línea. Confirmando la asumpción de Miles y Snow (1978) respecto a las características de los pioneros en innovación en la industria.

Situación similar, que valida los supuestos de estos autores, se encuentra respecto a las estrategias viables y la conducta fallida. Las organizaciones tienden a buscar una posición en la industria y por consiguiente eligen una postura que le permita lograrlo. No obstante, un rezago de empresas queda en movimiento desordenado por el espacio del mercado, optando por un comportamiento fallido a la merced del rendimiento que pueden lograr indirectamente (Anwar \& Hasnu, 2017); en este caso es sumamente baja la participación. 
Cabe aclarar que la anterior afirmación está sujeta al tipo de industria que se está analizando. Al ser un servicio educativo que está regulado por permiso gubernamental, los directivos pueden optar por mantener la licencia de funcionamiento emitida para solventar su inversión, antes de aventurar el desarrollo de productos. Esto a su vez puede traducirse en el número casi nulo de reactores.

Por otro lado, las capacidades que desarrollan las empresas son puntuadas en los intervalos superiores, y una pequeña fracción en nivel bajo para la capacidad tecnológica. Así pues, al contrario del foco sobre la operación del servicio para un mejor desarrollo de la organización (Day, 1994; Nieves \& Haller, 2014), las capacidades relacionadas con el mercado, tanto en marketing como en vínculos con los grupos de interés, son mayor apreciadas por estas compañías.

En tal sentido, la propuesto por Teece et al (1997) y Teece (2007) toma soporte de estos hallazgos. La entidad busca un mejor desempeño al actualizar sus capacidades conforme a los cambios que se presentan en el entorno, siendo el ambiente el antecedente para el ajuste interno (Danneels, 2010; Parnell \& Brady, 2019; Zander \& Kogut, 1995).

Trasladando esto a los resultados del clustering, las capacidades presentan variación entre las letdh. Diferentes niveles de desarrollo en cada una permiten generar la agrupación en tres clústeres con discrepancia entre ellos. Lo anterior permite afirmar la existencia de conductas estratégicas en las compañías, las cuales varían para mantener una postura frente a su mercado, y hacia la competencia (Desarbo \& Grewal, 2008). Sin embargo, sus capacidades no están alineadas totalmente a la estrategia descrita por la letdh.

Al respecto, hay una variación notable en el perfil y niveles que sigue cada uno de los clústeres (representado en la figura 3) especialmente entre el grupo 2 y los demás. La estrategia defensor, dominante para este último, agrupa una baja porción de las empresas analizadas, aunque está conducta es la de mayor representación en la muestra. Otra mayoría de esa estrategia tiene una puntuación mayor y se sitúan en el clúster 3. Por tanto, permite aducir la existencia de una postura híbrida entre defensor y analizador, pues su fuente de capacidad facilita a estas organizaciones trasladarse sobre el continuo de la defensa de nicho al desarrollo de productos rentables en el mercado (Anwar \& Hasnu, 2017; Claver-Cortés et al., 2012; Desarbo \& Grewal, 2008; Gabrielsson, Seppälä, \& Gabrielsson, 2016).

Para el clúster 1, la postura dominante es prospector, coincidiendo allí las empresas de esa conducta. Sin embargo, las capacidades de marketing y tecnológica puntúan semejante a los analizadores, y estos ocupan un espacio en el clúster. Esto reafirma el hallazgo anterior respecto al uso de estrategias combinadas en las compañías de servicios educativos; en este caso de tipo prospector - analizador.

Empero los hallazgos de perfiles en los grupos, el tipo de industria es relevante para su comprensión. Anwar y Hasnu $(2016,2017)$ encontraron incidencia del mercado de participación de la empresa, tanto en estrategias puras como híbridas, así como en posturas consistentes o flexibles respecto a su conducta; similares conclusiones plantean Desarbo y Grewal (2008), Claver-Cortés et al (2012), Parnell (2011) y Gabrielsson et al (2016).

Puede ser una situación de análisis ese hallazgo particular. Cuando existe limitación de desarrollo de productos a periodos específicos, por condiciones externas a la compañía, las organizaciones enfrentan un difícil salto entre los extremos de Miles y Snow (1978), lo cual hace de la postura intermedia un suave transito entre las estrategias opuestas.

\section{Conclusiones, implicaciones e investigación futura}

Este estudio investigó la afinidad que existe entre la postura estratégica descrita y aquella resultante de las capacidades organizacionales que mantienen las instituciones de Educación para el Trabajo y Desarrollo Humano 
en Colombia; extendiendo el conocimiento hacia un entorno aún no explorado con esas variables. Directivos en las empresas reportaron una mayor conducta defensora y analizadora que prospector, y casi nula la presencia de estrategia fallida reactor. Niveles medio y alto, con puntuaciones en el intervalo $(3,6]$, fueron identificados en cada compañía, para cinco capacidades organizacionales diferentes.

Si bien las capacidades fueron bien evaluadas, la referente a la operación del servicio alcanzó bajas puntuaciones, lo cual hace crítico la adecuada entrega de la propuesta de valor; pues las letdh enfocan sus esfuerzos en habilidades de actividad de marketing, más que en la forma de prestación del servicio. Esto se presenta por la naturaleza del mercado analizado. Sus productos tienen un corto ciclo de vida y mayor tasa de deserción del usuario, conllevando a una propensión del empresario por reposición continua de la base de clientes. Además, al someterse a licenciamiento por el gobierno local, implica un estándar mínimo de operación en cada producto.

El agrupamiento permitió encontrar el uso de posturas estratégicas híbridas, acorde a la base de capacidades que posee la empresa. Quizás este esfuerzo por fundamentación y conocimiento que buscan las letdh, contribuye a movimientos entre los extremos de la estrategia. Actuando como defensores cuando se ha obtenido permiso de operación de un nuevo producto, y procediendo como prospector cuando escasea la demanda de ese servicio, o cuando se quiere atender necesidades nuevas o cruzadas.

En cuanto a las implicaciones prácticas, los hallazgos plantean una descripción clara de las posturas dominantes en las empresas del mercado de letdh. El análisis de las capacidades permite a la organización direccionar sus esfuerzos de desarrollo de conocimiento hacia alguna de las perspectivas existentes en los grupos.

Además, el uso de la estrategia analizador puede ser utilizado como vía intermedia para desarrollar productos, a la vez que se cuida el mercado actual, sin caer en la no eficiencia de costos que tiende a encontrarse en la postura media. Los directivos podrán verificar sus condiciones internas, y optar por la estrategia que mejor logre la alineación de su mercado.

En lo metodológico, si bien se sugiere la existencia de problemas cuando constructos y encuestas son aplicados en contextos diferentes (Punnett \& Shenkar, 1994), los análisis de validación corroboran la adecuación de los instrumentos, tanto en estrategia como en capacidades, para una industria y entorno geográfico diferentes a los evaluados en la literatura.

No obstante, algunas limitaciones se presentan al referente. Factores externos al fenómeno como la cultura, relación social y ambiente de la empresa latinoamericana, pueden incidir en considerar las capacidades medidas como no importantes, o pueden existir otras que sean de mayor relevancia para el desarrollo competitivo de la empresa. Bajo tal situación, el alcance del estudio se limita a las habilidades evaluadas en las cinco variables latentes dispuestas.

Esto da cabida a oportunidades para futura investigación, analizando de manera exploratoria qué competencias mantienen las empresas colombianas y son utilizadas en su quehacer competitivo. Lo cual brindaría mayores luces respecto a la sujeción a determinado perfil estratégico, y ampliaría la base de capacidades a analizar en la relación de estas variables.

Los resultados del análisis arrojan otra luz de investigación. Mientras aquí la estrategia fue medida como posturas excluyentes entre ellas, puede ensancharse el estudio hacia una evaluación de conductas híbridas y puras. De igual manera, dadas las condiciones descritas de operación del mercado abordado, un segundo momento de valoración podría explicar la consistencia o flexibilidad del comportamiento estratégico.

Así mismo, el estudio no contempla una medida de performance. La literatura ha mostrado el concierto de lo interno y el ambiente de la empresa como el camino para el logro de beneficio en la organización. En tal sentido, 
futuros trabajos pueden incluir una valoración del rendimiento de la compañía, comprendiendo si esas capacidades en agrupaciones son portadoras de mayor excedente para esta.

Finalmente, la investigación cerró la muestra a un contexto mono-mercado, es decir, a empresas que actúan en un único dominio de actividad estratégica. Sería conveniente analizar en otras industrias, si el abordaje opera y permite el logro de resultados a comparar. Además, ampliar el análisis a plataformas de competencia multimercado, pues esa característica incide en la conducta que una compañía adopta en los dominios donde tiene presencia.

Son varias las vías de investigación que se plantean, tanto en la inclusión de nuevas variables, como en los momentos y contextos a ser replicado o extendido el estudio.

\section{Referencias bibliográficas}

Abell, D. F. (1980). Defining the Business: The Starting Point of Strategic Planning. Englewood Cliffs, New Jersey: Prentice-Hall.

Ambrosini, V., \& Bowman, C. (2009). What are dynamic capabilities and are they a useful construct in strategic management? International Journal of Management Reviews, 11(1), 29-49. https://doi.org/10.1111/j.1468-2370.2008.00251.x

Amit, R., \& Schoemaker, P. J. H. (1993). Strategic assets and organizational rent. Strategic Management Journal, 14(August), 33-46. https://doi.org/10.1002/smj.4250140105

Ansoff, I. (1979). Strategic Management. London: MacMillan.

Anwar, J., \& Hasnu, S. (2016). Business strategy and firm performance: a multi-industry analysis. Journal of Strategy and Management, 9(3), 361-382. https://doi.org/10.1108/JSMA-09-2015-0071

Anwar, J., \& Hasnu, S. A. F. (2017). Strategy-performance relationships: A comparative analysis of pure, hybrid, and reactor strategies. Journal of Advances in Management Research, 14(4), 446-465.

https://doi.org/10.1108/JAMR-07-2016-0056

Astrachan, C. B., Patel, V. K., \& Wanzenried, G. (2014). A comparative study of CB-SEM and PLS-SEM for theory development in family firm research. Journal of Family Business Strategy, 5(1), 116-128. https://doi.org/10.1016/j.jfbs.2013.12.002

Baez, D. Olazabal-Lugo, M. \& Romero-Muñoz, J. (2020). Toma de decisiones empresariales a través de la media ponderada ordenada. INQUIETUD EMPRESARIAL, 19(2), 11-23. Recuperado a partir de https://revistas.uptc.edu.co/index.php/inquietud_empresarial/article/view/9843

Barney, J. B. (1991). Firm Resources and Sustained Competitive Advantage. Journal of Management, 17(1), 99120. https://doi.org/10.1177/014920639101700108

Barney, J. B. (2002). Strategic management: from informed conversation to academic discipline. The Academy of Management Executive, 16(2), 53-57. https://doi.org/10.5465/AME.2002.7173521

Barney, J., Wright, M., \& Ketchen, D. J. (2001). The resource-based view of the firm Ten years after 1991. Journal of Management, 27(September), 625-641. https://doi.org/10.1177/014920630102700601

Blackmore, K., \& Nesbitt, K. (2013). Verifying the Miles and Snow strategy types in Australian small- and medium-size enterprises. Australian Journal of Management, 38(1), 171-190.

https://doi.org/10.1177/0312896212444692 
Bouhelal, F., \& Kerbouche, M. (2016). Why do we consider Miles and Snow's model's one of the most important strategic ones?. Maghreb Review of Economic and Management, 3(1), 23-34. https://doi.org/10.12816/0032860

Browne, M. W., \& Cudeck, R. (1992). Alternative ways of assessing model fit. Sociological Methods \& Research, 21(2), 230-258. https://doi.org/10.1177/0049124192021002005

Buzzell, R. D., Gale, B. T., \& Sultan, R. G. (1975). Market share - a key to profitability. Harvard Business Review, 53(1), 97-106.

Camisón Zornoza, C., Garrigós Simón, F. J., \& Palacios Marqués, D. (2007). Estrategias competitivas y desempeño empresarial: estudio comparativo de los modelos de Robinson \& Pearce y Miles \& Snow en el sector hotelero español. Investigaciones Europeas de Dirección y Economía de La Empresa, 13(3), 161-182.

Castro Monge, E. (2010). Las estrategias competitivas y su importancia en la buena gestión de las empresas. Ciencias Económicas, 28(1), 247-276.

Catena, A., Ramos, M. M., \& Trujillo, H. M. (2003). Análisis multivariado. Un manual para investigadores. Madrid: Biblioteca Nueva.

Chandler, A. D. (1962). Strategy and Structure. Cambridge: Massachusetts Institute of Technology Press.

Chernoff, H. (1973). The use of faces to represent points in k-dimensional space graphically. Journal of the American Statistical Association, 68(342), 361-368. https://doi.org/10.1080/01621459.1973.10482434

Child, J. (1972). Organizational structure, environment and performance: the role of strategic choice. Sociology, 6(1), 1-22. https://doi.org/10.1177/003803857200600101

Chryssochoidis, G., Dousios, D., \& Tzokas, N. (2016). Small Firm Adaptive Capability, Competitive Strategy, and Performance Outcomes: Competing Mediation vs Moderation Perspectives. Strategic Change, 25, 441466. https://doi.org/10.1002/jsc.2073

Claver-Cortés, E., Pertusa-Ortega, E. M., \& Molina-Azorín, J. F. (2011). Estructura organizativa y resultado empresarial: un análisis empírico del papel mediador de la estrategia. Cuadernos de Economía y Dirección de La Empresa, 14(1), 2-13. https://doi.org/10.1016/j.cede.2011.01.005

Claver-Cortés, E., Pertusa-Ortega, E. M., \& Molina-Azorín, J. F. (2012). Characteristics of organizational structure relating to hybrid competitive strategy: Implications for performance. Journal of Business Research, 65(7), 993-1002. https://doi.org/10.1016/j.jbusres.2011.04.012

Cohen, J. (1960). A coefficient of agreement for nominal scales. Educational and Psychological Measurement, 20(1), 37-46.

Conant, J. S., Mokwa, M. P., \& Varadarajan, P. R. (1990). Strategic types, distinctive marketing competencies and organizational performance: a multiple measures-based study. Strategic Management Journal, 11(5), 365-383.

Cyert, R. M., \& March, J. G. (1963). A Behavioral Theory of the Firm. Englewood Cliffs, New Jersey: Prentice-Hall.

Danneels, E. (2008). Organizational antecedents of second-order competences. Strategic Management Journal, 29(1), 519-543. https://doi.org/10.1002/smj.684

Danneels, E. (2010). Trying to become a different type of company: dynamic capability at Smith Corona. Strategic Management Journal, 32(April), 1-31. https://doi.org/10.1002/smj.863 
Day, G. S. (1994). The capabilities of market-driven organizations. Journal of Marketing, 58(4), 37-52. https://doi.org/10.4468/2008.2.06sciarelli

Desarbo, W. S., Di Benedetto, C. A., Song, M., \& Sinha, I. (2005). Revisiting the Miles and Snow strategic framework: Uncovering interrelationships between strategic types, capabilities, environmental uncertainty, and firm performance. Strategic Management Journal, 26(1), 47-74. https://doi.org/10.1002/smj.431

Desarbo, W. S., \& Grewal, R. (2008). Hybrid strategic groups. Strategic Management Journal, 29(3), 293-317. https://doi.org/10.1002/smj.658

Douglas, S. P., \& Rhee, D. K. (1989). Examining generic competitive strategy types in U.S. and european markets. Journal of International Business Studies, 20(3), 437-463.

Escobar-Pérez, J., \& Cuervo-Martínez, Á. (2008). Validez de Contenido y Juicio de Expertos: Una Aproximacón a su Utilización. Avances En Medición, 6(1), 27-36.

Farrukh, M., Meng, F., Wu, Y., \& Nawaz, K. (2020). Twenty-eight years of business strategy and the environment research: A bibliometric analysis. Business Strategy and the Environment, 29(6), 2572-2582. https://doi.org/ 10.1002/bse.2521

Fleiss, J. L. (1971). Measuring nominal scale among many raters. Psychological Bulletin, 76(5), 378-382. https://doi.org/10.1037/h0031619

Gabrielsson, M., Seppälä, T., \& Gabrielsson, P. (2016). Realizing a hybrid competitive strategy and achieving superior financial performance while internationalizing in the high-technology market. Industrial Marketing Management, 54, 141-153. https://doi.org/10.1016/j.indmarman.2015.07.001

Galbraith, C., \& Schendel, D. (1983). An empirical analysis of strategy types. Strategic Entrepreneurship Journal, 4(2), 153-173.

George, D., \& Mallery, P. (2016). IBM SPSS Statistics 23 Step by Step: A Simple Guide and Reference. IBM SPSS Statistics 25 Step by Step (14th ed.). New York: Taylor and Francis.

Grant, R. M. (1991). The resource-based theory of competitive advantage: implications for strategy formulation. California Management Review, 33(3), 114-135. https://doi.org/10.2307/41166664

Grant, R. M. (1996). Toward a knowledge-based theory of the firm. Strategic Management Journal, 17(Winter Spacial Issue), 109-122. https://doi.org/10.1002/smj.4250171110

Hair, J. F., Black, W. C., Babin, B. J., \& Anderson, R. E. (2019). Multivariate Data Analysis (8th ed.). Hamspshire: Cengage.

Herbert, T. T., \& Deresky, H. (1987). Generic strategies: an empirical investigation of typology validity and strategy content. Strategic Management Journal, 8(2), 135-147.

Hofer, C. W., \& Schendel, D. (1978). Strategy Formulation: Analytical Concepts. Minnesota: West Publishing Co.

Hyrkäs, K., Appelqvist-Schmidlechner, K., \& Oksa, L. (2003). Validating an instrument for clinical supervision using an expert panel. International Journal of Nursing Studies, 40(6), 619-625.

https://doi.org/10.1016/S0020-7489(03)00036-1 
Jusoh, R., \& Parnell, J. A. (2008). Competitive strategy and performance measurement in the Malaysian context: an exploratory study. Management Decision, 46(1), 5-31. https://doi.org/10.1108/00251740810846716

Kline, R. B. (2016). Principles and practices of structural equation modelling. Methodology in the social sciences (4th ed.). New York: The Guilford Press.

Kotler, P. (1992). Dirección de Marketing (7th ed.). Madrid: Prentice Hall.

Kotler, P., \& Keller, K. L. (2012). Dirección de Marketing (14th ed.). México: Pearson. https://doi.org/10.1017/CBO9781107415324.004

Landis, J. R., \& Koch, G. G. (1977). The Measurement of Observer Agreement for Categorical Data. Biometrics, $33(1), 159-174$.

Lin, C., Tsai, H.-L., \& Wu, J.-C. (2014). Collaboration strategy decision-making using the Miles and Snow typology. Journal of Business Research, 67(9), 1979-1990. https://doi.org/10.1016/j.jbusres.2013.10.013

Maia, J.L., Di Serio, L.C., \& Alves, A.G. (2015). Bibliometric Research on Strategy as Practice: Exploratory Results and Source Comparison. Sistemas y Gestao, 10(4), 654-669. https://doi.org/ 10.20985/1980-

5160.2015.v10n4.662

Maia, J.L., \& Di Serio, L.C. (2017). Corporate governance and business strategy: of bibliometric mapping the scientific production in the field. Revista Gestao \& Tecnologia-Journal of Management and Technology, 17(2), 160-185. https://doi.org/ 10.20397/2177-6652/2017.v17i2.1031

March, J. (1991). Exploration and Exploitation in Organizational Learning. Organization Science, 2(1), 71-87. https://doi.org/10.1007/sl

McGartland Rubio, D., Berg-Weger, M., Tebb, S. S., Lee, E. S., \& Rauch, S. (2003). Objectifyng content validity: Conducting a content validity study in social work research. Social Work Research, 27(2), 94-104. https://doi.org/10.1093/swr/27.2.94

Miles, R. H. (1982). Coffin nails and corporate strategies. Englewood Cliffs, New Jersey: Prentice-Hall.

Miles, R., \& Snow, C. (1978). Organizational Strategy, Structure, and Process. New York: McGraw-Hill.

Miller, D. (1987). Strategy making and structure: analysis and implications for performance. Academy of Management Journal, 30(1), 7-32.

Ministerio de Educación Nacional de Colombia. (2017). Resumen datos sistema de información de la educación para el trabajo y el desarrollo humano 2017. Retrieved December 1, 2017, from https://www.mineducacion.gov.co/1759/w3-article-353023.html

Mintzberg, H. (1988). Generic strategies: toward a comprehensive framework. In R. Lamb \& P. Shrivastava (Eds.), Advances in Strategic Management: a research annual. Greenwich: Jai Press Inc.

Mintzberg, H., Ahlstrand, B., \& Lampel, J. (2010). Safari a la estrategia. Buenos Aires: Granica.

Nieves, J., \& Haller, S. (2014). Building dynamic capabilities through knowledge resources. Tourism Management, 40, 224-232. https://doi.org/10.1016/j.tourman.2013.06.010 
O'Shannassy, T. (2008). Sustainable competitive advantage or temporary competitive advantage: improving understanding of an important strategy construct. Journal of Strategy and Management, 1(2), 168-180. https://doi.org/10.1108/17554250810926357

Parnell, J. A. (2011). Strategic capabilities, competitive strategy, and performance among retailers in Argentina, Peru and the United States. Management Decision, 49(1), 139-155. https://doi.org/10.1108/00251741111094482

Parnell, J. A. (2014). Strategic Management. Theory and practice. Ed. 4. Washington: Sage Publications.

Parnell, J. A., \& Hershey, L. (2005). The strategy-performance relationship revisited: the blessing and curse of the combination strategy. International Journal of Commerce and Management, 15(1), 17-33. https://doi.org/10.1108/10569210580000220

Parnell, J. A., Long, Z., \& Lester, D. (2015). Competitive strategy, capabilities and uncertainty in small and medium sized enterprises (SMEs) in China and the united states. Management Decision 53(2), 402-431. https://doi.org/10.1108/MD-04-2014-0222

Parnell, J., \& Brady, M. (2019). Capabilities, strategies and firm performance in the United Kingdom. Journal of Strategy and Management, (January). https://doi.org/10.1108/JSMA-10-2018-0107

Pituch, K. A., \& Stevens, J. P. (2016). Applied multivariate statistics for the social sciences: analyses with SAS and IBM's SPSS (6th ed.). New York: Routledge.

Porter, M. (2013). Estrategia competitiva. México: Patria.

Porter, M. E. (1980). Competitive Strategy. New York: The Free Press.

Punnett, B. J., \& Shenkar, O. (1994). International management research: toward a contingency approach. Advances in International Comparative Management, 9(1), 39-55.

Robinson, R. B., \& Pearce, J. A. (1988). Planned patterns of strategic behavior and their relationship to businessunit performance. Strategic Management Journal, 9(1), 43-60.

Segev, E. (1987a). A detailed methodological appendix for: Strategy, size and performance - an empirical investigation. Management Science, 33(2), 258-269. https://doi.org/10.1287/mnsc.33.2.258

Segev, E. (1987b). Strategy, strategy-making, and performance in a business game. Strategic Management Journal, 8(6), 565-577.

Skjong, R., \& Wentworth, B. H. (2000). Expert Judgement and Risk Perception. Det Norske Veritas, 2(1), 1-8.

Suárez Hernández, J., \& Ibarra Mirón, S. (2002). La teoría de los recursos y las capacidades: un enfoque actual en la estrategia empresarial. Anales de Estudios Económicos y Empresariales, (15), 63-89.

Summers, B., Williamson, T., \& Read, D. (2004). Does method of acquisition affect the quality of expert judgment? A comparison of education with on-the-job learning. Journal of Occupational and Organizational Psychology, 77(2), 237-258. https://doi.org/10.1348/096317904774202162

Teece, D. J. (2007). Explicating dynamic capabilities: the nature and microfoundations of (sustainable) enterprise performance. Strategic Management Journal, 28(August), 1319-1350. https://doi.org/10.1002/smj.640 
Teece, D. J., Pisano, G., \& Shuen, A. (1997). Dynamic capabilities and strategic management: organizing for innovation and growth. Strategic Management Journal, 18(7), 509-533.

Thompson, J. D. (1967). Organizations in Action. New York: McGraw-Hill.

Torres-Barreto, M., Noel, J., Meza-Ariza, L. \& Muñoz, L. (2016). El cambio tecnológico en el caso de los textiles inteligentes: Una aproximación desde las capacidades dinámicas. Revista Espacios, 37(8), 12-26. https://www.revistaespacios.com/a16v37n08/16370813.html

Treacy, M., \& Wiersema, F. (1997). The Discipline of market leaders. New York: Basic Books.

Utterback, J. M., \& Abernathy, W. J. (1975). A dynamic model of process and product innovation. OMEGA, 3(6), 639-656. https://doi.org/10.1016/0305-0483(75)90068-7

Vesper, V. D. (1979). Strategic mapping - a tool for corporate planners. Long Range Planning, 12(6), 75-92. https://doi.org/10.1016/0024-6301(79)90059-1

Vogel, R., \& Güttel, W. H. (2013). The dynamic capability view in strategic management: a bibliometric review. International Journal of Management Reviews, 15(4), 426-446. https://doi.org/10.1111/ijmr.12000

Wang, C. L., \& Ahmed, P. K. (2007). Dynamic capabilities: A review and research agenda. International Journal of Management Reviews, 9(1), 31-51. https://doi.org/10.1111/j.1468-2370.2007.00201.x

Wissema, J. G., Van Der Pol, H. W., \& Messer, H. M. (1980). Strategic management archetypes. Strategic Management Journal, 1(1), 37-47. https://doi.org/10.1002/smj.4250010105

Witten, I. H., Frank, E., Hall, M. A., \& Pal, C. J. (2017). Data Mining. Practical Machine Learning Tools and Techniques (4th ed.). Cambridge: Morgan Kaufmann.

Worthington, R. L., \& Whittaker, T. A. (2006). Scale development research: A content analysis and recommendations for best practices. The Counseling Psychologist, 34(6), 806-838. https://doi.org/10.1177/0011000006288127

Ynzunza, C. B., \& Izar, J. M. (2013). Efecto de las estrategias competitivas y los recursos y capacidades orientados al mercado sobre el crecimiento de las organizaciones. Contaduría y Administración, 58(1), 169-197. https://doi.org/http://dx.doi.org/10.1016/S0186-1042(13)71202-6

Zahra, S. A., Sapienza, H. J., \& Davidsson, P. (2006). Entrepreneurship and dynamic capabilities: A review, model and research agenda. Journal of Management Studies, 43(4), 917-955. https://doi.org/10.1111/j.14676486.2006.00616.x

Zander, U., \& Kogut, B. (1995). Knowledge and the speed of the transfer and imitation of organizational capabilities: an empirical test. Organization Science, 6(1), 76-92. https://doi.org/10.1287/orsc.6.1.76

\section{Anexo 1. Instrumento de medición para estrategia competitiva}

\section{Estrategia defensor:}

1. La empresa intenta localizar un nicho seguro en una mezcla de productos relativamente estable.

2. La empresa intenta mantener un nicho seguro en una mezcla de productos relativamente estable.

3. La empresa tiende a ofrecer un conjunto de productos más estrecho que sus competidores. 
4. La empresa trata de proteger su porción del mercado en el que opera, al ofrecer una mayor calidad que sus competidores.

5. La empresa trata de proteger su porción del mercado en el que opera, al ofrecer precios más bajos que sus competidores.

6. La empresa se concentra en tratar de lograr el mejor rendimiento en un dominio producto-mercado relativamente estrecho.

7. La empresa pone menos énfasis en el análisis de los cambios en la industria, que no son directamente relevantes a ella.

8. La empresa trata de proteger su porción del mercado en el que opera, al ofrecer un mejor servicio que sus competidores.

9. La empresa no es un líder de innovación y desarrollo en la industrla.

\section{Estrategia prospector:}

1. La empresa es reconocida en la industria por ser innovadora y creativa.

2. La empresa opera con una mezcla de productos amplia (muchos programas académicos) con respecto a sus competidores.

3. La empresa se esfuerza en ser de los primeros en desarrollar nuevos productos (o mercados geográficos) dentro de su industria.

4. No todos los esfuerzos que ha hecho la empresa, para ser "el primero en" desarrollo de productos dentro de su industria, han sido rentables.

5. La empresa responde rápidamente a señales tempranas de oportunidades en el mercado.

6. Las acciones de la empresa a menudo conllevan a una nueva ronda de competencia en la industria.

7. Al responder a todas las oportunidades de mercado, la empresa no es capaz de mantener la fuerza de todas sus líneas de producto en el mercado que opera.

8. La mezcla de productos es redefinida periódicamente.

Estrategia analizador:

1. Además de los productos estables, la empresa adopta rápidamente las innovaciones prometedoras en la industria.

2. La empresa intenta mantener una línea limitada de productos.

3. La empresa intenta mantener una línea estable de productos.

4. Las innovaciones seleccionadas por la empresa son evaluadas cuidadosamente.

5. La empresa mantiene un control ciudadoso de sus costos cuando genera nuevos productos para el mercado.

6. La empresa monitorea en detalle las acciones de los competidores en las áreas que se parecen más a la mezcla estable de producto/mercado que posee.

7. La empresa pocas veces desarrolla nuevos productos e innovaciones en la industria.

8. La empresa con frecuencia es capaz de desarrollar rápidamente un producto o servicio, tan efectivo y rentable, de los que ha introducido su principal competidor. 


\section{Estrategia reactor:}

1. La empresa no es agresiva para defender su dominio de producto/mercado, en comparación con lo que hacen sus competidores en la industria.

2. La empresa trata de no tomar muchos riesgos como lo hacen algunos de sus competidores.

3. La empresa reacciona en las áreas en las que su entorno ejerce presión sobre ella o amenaza su dominio.

4. La empresa planifica su actuar acorde se vayan presentando dificultades en el mercado.

5. Aunque la empresa tiene una oferta de productos/mercados, aún no ha definido una orientación consistente de ellos.

6. La empresa monitorea el mercado de manera esporádica o casi nunca.

\section{Anexo 2. Instrumento de medición para capacidades organizacionales}

\section{Capacidades de marketing:}

1. Conocimiento de los clientes

2. Conocimiento de los competidores

3. Integración de las actividades de mercadeo

4. Habilidad para segmentar y determinar mercados objetivo

5. Efectividad de los programas de precio

6. Efectividad de los programas de publicidad

Capacidades de enlace de mercado:

1. Capacidades de detección de mercado

2. Creación y gestión de relaciones duraderas con clientes

3. Capacidad de crear relaciones duraderas con los proveedores

4. Habilidad para retener clientes

5. Capacidad de enlace con los miembros del canal

6. Relaciones con los miembros del canal

Capacidades de tecnología de información:

1. Sistemas de información para proyectos de desarrollo de nuevos productos

2. Sistemas de información para facilitar la integración interfuncional (integración entre los procesos y áreas de la empresa)

3. Sistemas de información para facilitar la creación de conocimientos tecnológicos

4. Sistemas de información para facilitar la creación de conocimiento de mercado

5. Sistemas de información para la comunicación interna (p.e., a lo largo de diferentes departamentos, de diferentes niveles de la organización, etc.)

6. Sistemas de información para la comunicación externa (p.e., proveedores, clientes, miembros del canal, etc.) 
Capacidades tecnológicas:

1. Capacidad de desarrollar nuevos productos

2. Procesos de fabricación/servucción

3. Capacidades de desarrollo de tecnología

4. Habilidad de predecir cambios tecnológicos en la industria

5. Instalaciones para producción

6. Habilidades de variar la calidad (control de calidad)

Capacidades de gestión:

1. Sistemas logísticos integrados

2. Capacidades de control de costos

3. Habilidades de gestión financiera

4. Capacidades de gestión de recursos humanos

5. Precisión del pronóstico de rentabilidad e ingresos

6. Proceso de planificación de marketing

7. Proceso de planificación estratégica de la compañía

8. Evaluación periódica del cumplimiento de la estrategia y objetivos

Esta obra está bajo una Licencia Creative Commons

Attribución-NoCommercial 4.0 International

\section{(cc) EY-NC}

